\title{
铜催化炔基溴与丙炔醇的偶联-异构化反应合成戊炔烯酮
}

\author{
${\text { 杨宗莲 }{ }^{a} \text { 廖沛球 }}^{a} \quad$ 李兴奇 ${ }^{a}$ 毕锡和 $*, a, b$ \\ $\left({ }^{a}\right.$ 东北师范大学化学学院 长春 130024$)$ \\ ( ${ }^{b}$ 南开大学元素有机化学国家重点实验室 天津 300071)
}

\begin{abstract}
摘要 乙炔澳和丙炔醇在哌啶溶剂中, 用醋酸铜作催化剂, 能够发生偶联-异构化反应获得具有立体选择性的唯一产 物 $(E)$-1,5-二取代-戊-2-烯-4-炔-1-酮. 机理研究表明, 哌啶在反应体系中起到了溶剂和碱的双重作用. 该反应具有简单、 高效、反应范围宽泛等特点，能够以 $40 \% \sim 83 \%$ 的产率获得目标产物反式 1,5 -二取代的戊炔烯酮.

关键词 戊炔烯酮; 乙炔澳; 丙炔醇; 偶联-异构化反应
\end{abstract}

\section{Pentynenones Formation through Copper Catalyzed Coupling- Isomerization Reaction between Alkynyl Bromides and Propargylic Alcohols}

\author{
Yang, Zonglian ${ }^{a}$ \\ Liao, Peiqiu ${ }^{a}$ \\ Li, Xingqi ${ }^{a}$ \\ $\mathrm{Bi}, \mathrm{Xihe}^{*, a, b}$ \\ $\left({ }^{a}\right.$ Department of Chemistry, Northeast Normal University, Changchun 130024) \\ $\left({ }^{b}\right.$ State Key Laboratory of Elemento-Organic Chemistry, Nankai University, Tianjin 300071)
}

\begin{abstract}
Disubstituted (E)-pent-2-en-4-yn-1-ones can be exclusively synthesized by copper-catalyzed tandem coupling-isomerization reaction of alkynyl bromides and secondary propargylic alcohols in piperidine. This method is operation-simple and allows the synthesis of a wide variety of stereodefined 1,5-disubstituted pentynenones in $40 \% \sim 83 \%$ yields. The mechanistic investigations suggest that piperidine plays a duel role being both a solvent and a base in the reaction. Keywords pentynenones; alkynyl bromides; propargylic alcohols; coupling-isomerization reaction
\end{abstract}

戊炔烯酮是含有共轭 $\mathrm{C} \equiv \mathrm{C}, \mathrm{C}=\mathrm{C}$ 和 $\mathrm{C}=\mathrm{O}$ 键的一 类化合物. 该类化合物广泛存在于天然产物中, 是具有 潜在生物活性的一类化合物. 同时，由于戊炔烯酮含有 参键、双键和酮羰基相互共轭的结构特征，其不但具有 孤立炔烃、烯烃和酮羰基的基本性质，同时还具有由共 轭相互作用所带来的独特性质, 是一类非常重要的有机 合成中间体 ${ }^{[1 \sim 5]}$. 这些官能化的共轭炔烯酮, 能够被选 择性地修改用以合成许多杂环化合物，如呋喃 ${ }^{[1]}$ 、吡 唑 ${ }^{[2]}$ 、吡喃 ${ }^{[3]}$ 、二氢硫代吡喃酮 $[3,3 \mathrm{c}, 4]$ 和天然产物或药物 全合成中的分子片段 ${ }^{[5]}$. 因此，戊炔烯酮的合成方法引 起了大量合成研究者的兴趣, 并发展了许多新的合成方 法来合成该类化合物. 经过文献调研, 我们发现目前普 遍使用的合成方法是利用炔烃和炔烃衍生物作为反应 前体来获得炔烯酮化合物, 尤其是 1,5-二取代戊炔烯酮
类化合物 ${ }^{[69]}$. 如图 1 所示, 所用方法包括: (1)碱催化 1,5-二取代戊二炔基化合物的甲硅烷基醚或它们的醇衍 生物的异构化反应 ${ }^{[6]} ;(2)$ 末端炔烃和烯烃之间的偶联反 应 ${ }^{[7]} ;(3)$ 芳炔醛和芳基甲基酮的克莱森-施密特缩合反 应 $^{[8]}$; (4) Pd-催化的叔炔丙醇和烯烃的炔化反应 ${ }^{[9]}$. 此 外，合成含有多个取代基的戊炔烯酮的方法也多有报 道，比如芳基炔丙基甲基醚的烷基化反应 ${ }^{[10]}$, 端炔与活 化烯酮或烯醛的 Sonogashira 偶联反应 ${ }^{[11]}$ ，以及丙炔醛 的缩合反应 ${ }^{[12]}$ 等. 然而, 这些方法有的会产生大量副产 物给环境带来危害 ${ }^{[7]}$, 有的会导致生成炔烯酮的 $Z / E$ 两 种异构体的混合物 ${ }^{[6,10]}$. 因此, 发展能够简单、高效地获 得具有立体选择性的戊炔烯酮的合成方法是非常必要 的. 炔丙醇是一类易得的双官能化合成砌块，近年来， 在有机合成中被广泛应用 ${ }^{[13]}$. 在各种碱催化条件下, 通

\footnotetext{
*E-mail: bixh507@nenu.edu.cn

Received November 10, 2010; revised December 16, 2014; published online December 18, 2014.

Project supported by the National Natural Science Foundation of China (Nos. 21172029, 21202016, 21372038).

国家自然科学基金(Nos. 21172029, 21202016, 21372038)资助项目.
} 
过炔丙醇或炔丙醇甲硅烷基醚的异构化反应能够获得 反式或顺式的查尔酮衍生物 ${ }^{[14]} .2010$ 年, 刘元红课题 组 ${ }^{[6]}$ 报道了碱催化 1,5 -二取代戊二炔基化合物的甲硅烷 基醚或醇的异构化反应，能够高选择性地获得顺式或反 式的 1,5-二取代戊炔烯酮类化合物, 但所得产物中仍然 会有少量的 $Z / E$ 异构体的混合物存在. 我们课题组 ${ }^{[15]}$ 在 发展基于官能化炔烃的新反应基础上，发现炔基溴化物 和端基炔丙醇在醋酸铜催化下，可以发生交叉偶联反应 生成 1,5-二取代的戊二炔醇, 获得的戊二炔醇在哌啶溶 剂条件下能够进一步异构化、选择性专一的生成反式共 轭戊炔烯酮. 该反应中加入了金属铜催化剂, 反应机理 与只有碱催化的戍二炔醇异构化反应不同 ${ }^{[6]}$, 反应体系 中生成一种反式的戊炔烯酮化合物. 因此, 本文报道一 种反应条件温和、高效地铜催化一锅法合成反式 1,5-二 取代戊炔烯酮的新反应. 在哌啶溶剂中使用 $\mathrm{Cu}(\mathrm{OAc})_{2}$ 为催化剂, 氮气保护下, $0{ }^{\circ} \mathrm{C}$ 条件下催化炔基溴化物和 炔丙醇反应 $1 \mathrm{~h}$, 之后升温到常温下反应 $12 \mathrm{~h}$, 能够获得 具有立体选择性的 $(E)-1,5$-二取代-戊-2-烯-4-炔-1-酮. 该反应中，哌啶既是溶剂，同时也是碱性介质。

\section{1 结果与讨论}

\section{1 反应条件优化}

首先, 以 4-氯苯基乙炔溴和苯基丙炔醇为模型反应 底物，在 $\mathrm{N}_{2}$ 保护条件下，考察了各类铜盐及其他金属催
化剂、溶剂对反应结果的影响，结果见表 1 . 当使用 $\mathrm{Cu}(\mathrm{OAc})_{2}$ 为催化剂时, 通过对溶剂的笁选, 发现溶剂对 反应的进行有显著影响. 例如，使用 1,8-二氮杂双环 [5.4.0]十一碳-7-烯(DBU)作为溶剂, 产物产率只有 $16 \%$ (表 1, Entry 1). 而其他溶剂如吗啉、1,4-二氮杂二环 [2.2.2]辛烷(DACBO)和 $N$-甲基哌啶都不能使反应进行 (表 1, Enries 2 4). 使用哌啶为溶剂的反应效果最好, 产物 3a 产率升高到 82\%(表 1, Entry 5). 然后，我们考察 了一价铜盐如 $\mathrm{CuI}$ 和 $\mathrm{CuCl}$ 的催化效果, 发现一价铜盐 催化效果没有二价铜盐好，只分别获得了 $31 \%$ 和 $65 \%$ 的 产率(表 1, Entries 6 7). 其他的金属催化剂如 $\mathrm{Pb}\left(\mathrm{PPh}_{3}\right)_{2}$ 和碳酸银的催化效果也并不令人满意, $\mathrm{Pb}\left(\mathrm{PPh}_{3}\right)_{2}$ 不能使 反应发生(表 1 , Entry 8 ), 而使用碳酸银只能得到产率为 $12 \%$ 的产物(表 1, Entry 9). 最后, 为了研究哌啶在该反 应中是否起到了溶剂和碱的双重作用, 我们将哌啶的量 减少到 $1.3 \mathrm{~mL}$, 同时在反应体系中加入另外一种溶剂. 然而，所得的反应结果均没有单一使用哌啶溶剂的好. 在哌啶和 THF 的混合溶剂下能获得 $50 \%$ 产率的产物(表 1, Entry 10), 其他溶剂如甲苯、DMF 和 1,4-二氧六环与 哌啶的混合溶剂均不能使反应发生(表 1, Entries 11 13). 条件对照实验表明反应在空气中进行时产物产率 只有 $53 \%$ ，表明该反应采用 $\mathrm{N}_{2}$ 气保护是必要的(表 1 , Entry 14).

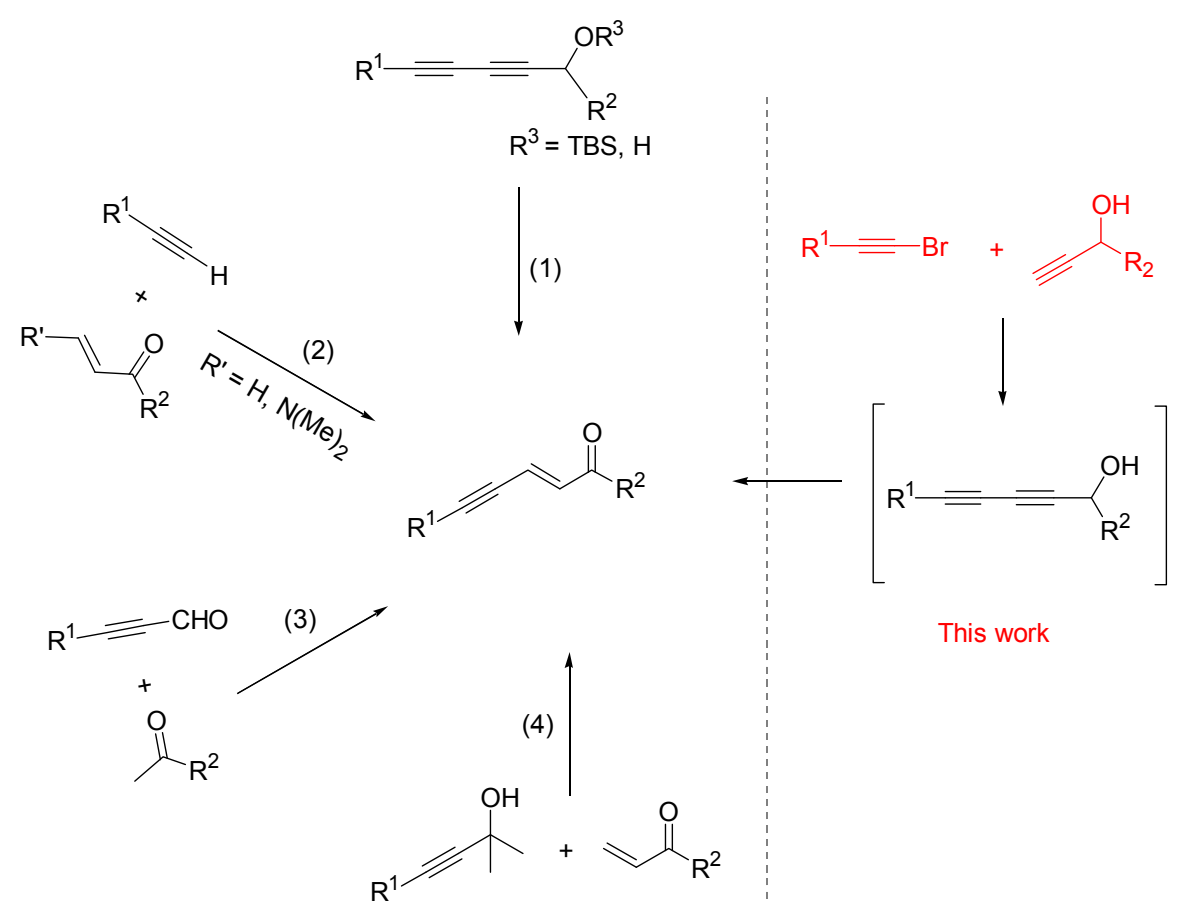

图 1 合成 1,5-二取代戊炔烯酮的方法

Figure 1 Strategies for the construction of 1,5-disubstituted pentynenones 
表 1 反应条件篮选和优化 ${ }^{a}$

Table 1 Reaction conditions screening and optimization

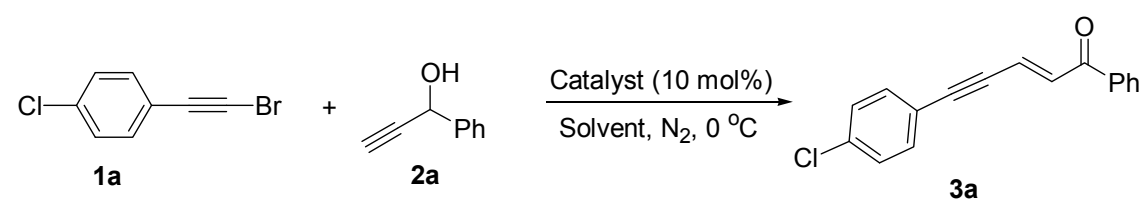

$1 \mathrm{a}$

2

\begin{tabular}{|c|c|c|c|c|}
\hline Entry 1 & Catalyst (equiv.) & Solvent $1(\mathrm{~mL})$ & Solvent 2 (mL) & $\mathrm{Yield}^{b} / \%$ \\
\hline 1 & $\mathrm{Cu}(\mathrm{OAc})_{2}(0.1)$ & $\mathrm{DBU}(2.0)$ & - & 16 \\
\hline 2 & $\mathrm{Cu}(\mathrm{OAc})_{2}(0.1)$ & Morpholine (2.0) & - & n.r. \\
\hline 3 & $\mathrm{Cu}(\mathrm{OAc})_{2}(0.1)$ & $\mathrm{DABCO}(2.0)$ & - & n.r. \\
\hline 4 & $\mathrm{Cu}(\mathrm{OAc})_{2}(0.1)$ & N-Methylpiperidine (2.0) & - & n.r. \\
\hline 5 & $\mathrm{Cu}(\mathrm{OAc})_{2}(0.1)$ & Piperidine $(2.0)$ & - & 82 \\
\hline 6 & $\mathrm{CuI}(0.1)$ & Piperidine (2.0) & - & 31 \\
\hline 7 & $\mathrm{CuCl}(0.1)$ & Piperidine (2.0) & - & 65 \\
\hline 8 & $\mathrm{~Pb}\left(\mathrm{PPh}_{3}\right)_{2}(0.1)$ & Piperidine (2.0) & - & n.r. \\
\hline 9 & $\mathrm{Ag}_{2} \mathrm{CO}_{3}(0.1)$ & Piperidine (2.0) & - & 12 \\
\hline 10 & $\mathrm{Cu}(\mathrm{OAc})_{2}(0.1)$ & Piperidine (1.3) & THF $(0.7)$ & 50 \\
\hline 11 & $\mathrm{Cu}(\mathrm{OAc})_{2}(0.1)$ & Piperidine (1.3) & Toluene $(0.7)$ & n.r. \\
\hline 12 & $\mathrm{Cu}(\mathrm{OAc})_{2}(0.1)$ & Piperidine (1.3) & $\operatorname{DMF}(0.7)$ & n.r. \\
\hline 14 & $\mathrm{Cu}(\mathrm{OAc})_{2}(0.1)$ & Piperidine (2.0) & - & $53^{c}$ \\
\hline
\end{tabular}

${ }^{a}$ 反应条件: 4 -氯苯基乙炔溴 $(0.5 \mathrm{mmol})$, 苯基丙炔醇 $(0.65 \mathrm{mmol})$, 催化剂 $(0.05 \mathrm{mmol})$, 溶剂 $(2.0 \mathrm{~mL}) ;$ 反应时间 $13 \mathrm{~h} .{ }^{b}$ 分离产率; ${ }^{c}$ 空气中.

\section{2 铜催化不同底物合成 1,5-二取代戊炔烯酮}

利用 $\mathrm{Cu}(\mathrm{OAc})_{2}$ 催化条件下 4 -氯苯基乙炔溴和苯基 丙炔醇反应合成反式 1,5 -二取代戊炔烯酮的最佳条件 (表 1, Entry 5)后，通过改变乙炔基溴化物的 $\mathrm{R}^{1}$ 基团和丙 炔醇的 $\mathrm{R}^{2}$ 基团, 我们进一步研究了此催化体系的普适 性(表 2). 从表 2 可知, 催化体系对于各种乙炔溴和丙炔 醇均具有较好的催化活性, 对于大部分底物均能以较好 收率专一地生成相应目标产物. 首先我们将各种不同取 代基的乙炔溴与苯基炔丙醇 $\mathbf{2 a}$ 进行反应. 如表 2 所示, 取代基官能团如芳基、杂芳基、稠芳基、烷氧基和正丁 基等均可与苯基炔丙醇反应, 以较高的产率获得反式戊 炔烯酮化合物(表 2, Entries 1 9). 对 $\mathrm{R}^{1}$ 基团的电子效 应进行研究后, 发现供电子基团和吸电子基团的乙炔溴 与苯基炔丙醇反应无显著取代基效应，都能生成相应的 反式戊炔烯酮, 产率从 $40 \% \sim 83 \%$. 为了扩大这种方法 的底物适用范围, 我们研究了具有不同 $\mathrm{R}^{2}$ 取代基的丙 炔醇与 4-氯苯基乙炔溴的反应. 实验表明, 含有不同取 代基的丙炔醇同样表现出良好的反应活性. 富电子或缺 电子的 $\mathrm{R}^{2}$ 取代基对该反应所得产物产率没有显著影响 (表 2, Entries $10 \sim 17$ ). 例如, 当 $\mathrm{R}^{2}$ 的取代基为 4- $-\mathrm{MeC}_{6} \mathrm{H}_{4}, 3-\mathrm{CF}_{3} \mathrm{C}_{6} \mathrm{H}_{4}, 2$-䒺基和 2-噻吩基时, 产物产率 较高 $(72 \% \sim 81 \%$, 表 2 , Entries $10 \sim 11,15,17)$; 当 $\mathrm{R}^{2}$ 取 代基为 2,4- $\mathrm{Cl}_{2} \mathrm{C}_{6} \mathrm{H}_{4}$ 、反式的 $\mathrm{PhCH}=\mathrm{CH}$ 、胡椒基和 3吡啶基时, 产物产率相对偏低, 为 41\% 63\%(表 2, Entries 12 14,16). 当 $\mathrm{R}^{2}$ 基团为空间位阻比较大的环已基 时, 没有获得目标产物 1,5-二取代戊炔烯酮, 而是产率
为 74\%的 1,5-二取代戊二炔醇 3s(表 2, Entry 18). 由此可 见, 乙炔溴与丙炔醇在铜催化下是先通过偶联反应生成 戊二炔醇后再进一步异构化生成了目标产物戊炔烯酮. 反式戊炔烯酮的结构经过 ${ }^{1} \mathrm{H} N \mathrm{NM},{ }^{13} \mathrm{C}$ NMR, HRMS 和 $X$ 射线衍射(3r)分析方法的确认(图 2).

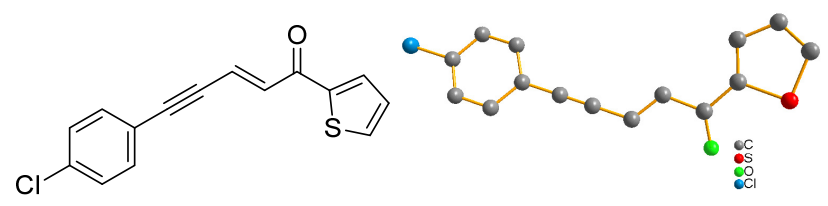

图 $23 r$ 的晶体结构

Figure 2 The crystal structure of $\mathbf{3 r}$

\section{3 反应机理分析}

根据炔溴和端炔化合物在铜和碱催化下容易发生 偶联反应的特点 ${ }^{[16]}$ 以及该反应得到的产物, 我们推测 反应可能经历以下过程(图 3): 首先乙炔溴与端基炔丙 醇发生交叉偶联反应生成 1,5-二取代的戊二炔醇 $\mathbf{A}$, 然 后哌啶分子和被铜活化的 $\mathrm{C} \equiv \mathrm{C}$ 参键发生亲核加成, 产 生中间体 $\mathbf{C}, \mathbf{C}$ 从溶剂(Piperidine) 中获取质子后转化成 炔烯醇中间体 $\mathbf{D}, \mathbf{D}$ 在哌啶碱作用下发生 1,3-氢迁移生 成中间体 $\mathbf{E}, \mathbf{E}$ 不稳定，能够很容易的通过酮-烯醇互变 异构产生化合物 $\mathbf{F}$, 化合物 $\mathbf{F}$ 可以从反应体系中分离出 来, 且化合物 $\mathbf{F}$ 通过酸性环境的硅胶柱层析后哌啶基离 去，可以得到目标化合物 3 . 
表 2 1,5-二取代戊炔烯酮的合成 ${ }^{a}$

Table 2 The synthesis of 1,5-disubstituted pentynenones

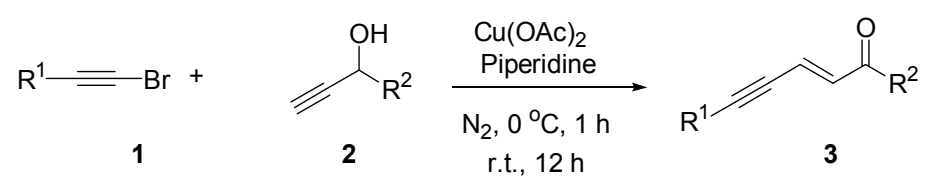

\begin{tabular}{|c|c|c|c|c|}
\hline Entry & $\mathrm{R}^{1}$ & $\mathrm{R}^{2}$ & 3 & Yield $^{b} / \%$ \\
\hline 1 & $\mathrm{Ph}(\mathbf{1 b})$ & $\mathrm{Ph}(\mathbf{2 a})$ & $3 \mathbf{b}$ & 50 \\
\hline 2 & $4-\mathrm{MeOC}_{6} \mathrm{H}_{4}(\mathbf{1 c})$ & $\mathrm{Ph}(\mathbf{2 a})$ & $3 \mathbf{c}$ & 72 \\
\hline 3 & $3-\mathrm{MeOC}_{6} \mathrm{H}_{4}(\mathbf{1 d})$ & $\mathrm{Ph}(\mathbf{2 a})$ & 3d & 60 \\
\hline 4 & $4-\mathrm{FC}_{6} \mathrm{H}_{4}(\mathbf{1 e})$ & $\mathrm{Ph}(\mathbf{2 a})$ & $3 \mathbf{e}$ & 83 \\
\hline 5 & 2-Naphthyl (1f) & $\mathrm{Ph}(\mathbf{2 a})$ & $3 f$ & 78 \\
\hline 6 & 2-Thienyl (1g) & $\mathrm{Ph}(\mathbf{2 a})$ & $3 g$ & 52 \\
\hline 7 & $2-\mathrm{BrC}_{6} \mathrm{H}_{4} \mathrm{OCH}_{2}(\mathbf{1 h})$ & $\mathrm{Ph}(\mathbf{2 a})$ & $3 \mathrm{~h}$ & 42 \\
\hline 8 & $\mathrm{PhCOOCH}_{2}(\mathbf{1 i})$ & $\mathrm{Ph}(\mathbf{2 a})$ & $3 \mathbf{i}$ & 50 \\
\hline 9 & $N$-Butyl & $\mathrm{Ph}(\mathbf{2 a})$ & $3 \mathbf{j}$ & 40 \\
\hline 10 & $4-\mathrm{ClC}_{6} \mathrm{H}_{4}(\mathbf{1 a})$ & $4-\mathrm{MeC}_{6} \mathrm{H}_{4}(\mathbf{2 b})$ & $3 \mathbf{k}$ & 81 \\
\hline 11 & $4-\mathrm{ClC}_{6} \mathrm{H}_{4}(\mathbf{1 a})$ & $3-\mathrm{CF}_{3} \mathrm{C}_{6} \mathrm{H}_{4}(\mathbf{2 c})$ & 31 & 72 \\
\hline 12 & $4-\mathrm{ClC}_{6} \mathrm{H}_{4}(\mathbf{1 a})$ & $2,4-\mathrm{Cl}_{2} \mathrm{C}_{6} \mathrm{H}_{3}(\mathbf{2 d})$ & $3 m$ & 61 \\
\hline 13 & $4-\mathrm{ClC}_{6} \mathrm{H}_{4}(\mathbf{1 a})$ & trans $-\mathrm{PhCH}=\mathrm{CH}(\mathbf{2 e})$ & $3 n$ & 41 \\
\hline 14 & $4-\mathrm{ClC}_{6} \mathrm{H}_{4}(\mathbf{1 a})$ & Piperonyl (2f) & 30 & 52 \\
\hline 15 & $4-\mathrm{ClC}_{6} \mathrm{H}_{4}(\mathbf{1 a})$ & 2-Naphthyl (2g) & $3 p$ & 78 \\
\hline 16 & $4-\mathrm{ClC}_{6} \mathrm{H}_{4}(\mathbf{1 a})$ & 3-Pyridyl (2h) & $3 q$ & 63 \\
\hline 17 & $4-\mathrm{ClC}_{6} \mathrm{H}_{4}(\mathbf{1 a})$ & 2-Thienyl (2i) & $3 \mathbf{r}$ & 75 \\
\hline 18 & $4-\mathrm{ClC}_{6} \mathrm{H}_{4}(\mathbf{1 a})$ & Cyclohexyl (2j) & $3 s$ & 74 \\
\hline
\end{tabular}

$\bar{a}$ 反应条件: 乙炔溴 $(0.5 \mathrm{mmol})$, 丙炔醇 $(0.65 \mathrm{mmol})$, 醋酸铜 $(0.1 \mathrm{mmol})$, 哌啶 $(2.0 \mathrm{~mL}) ;{ }^{b}$ 分离产率.
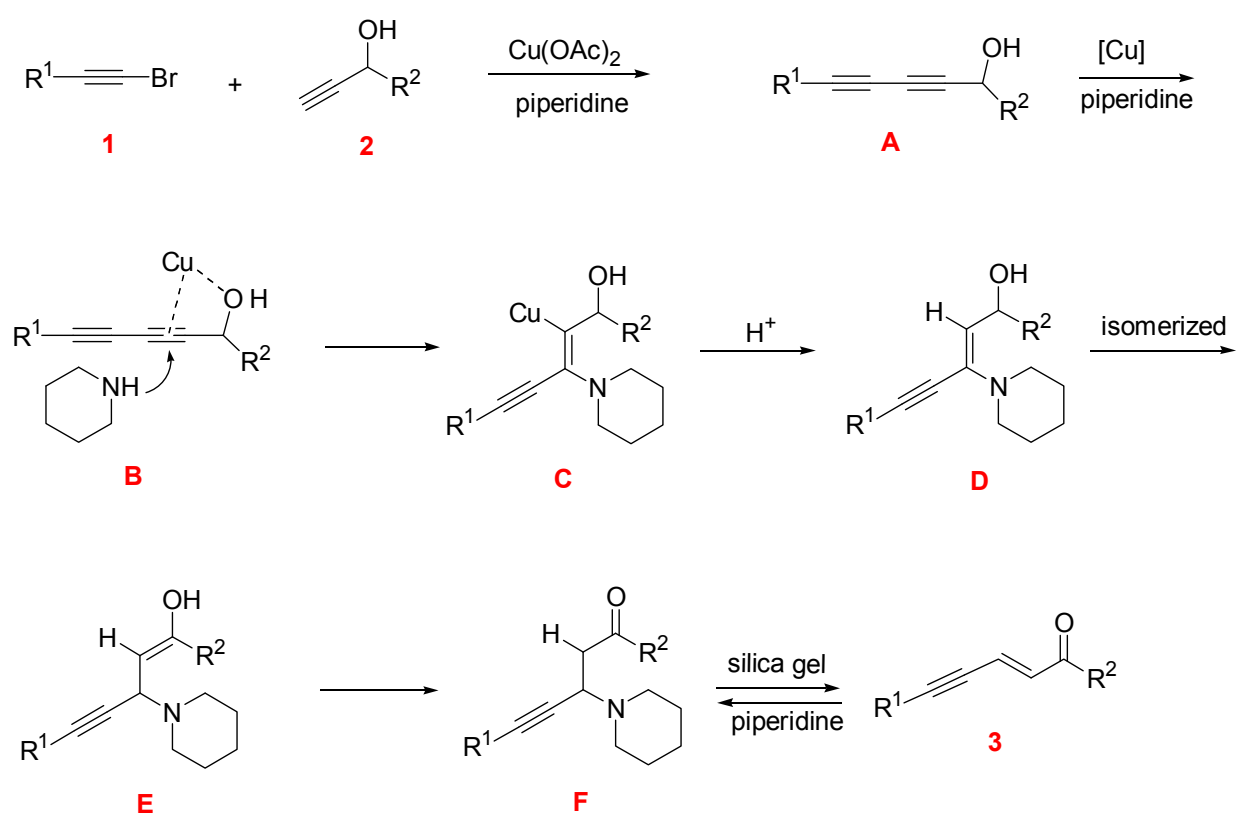

图 3 可能的催化机理

Figure 3 The possible catalytic mechanism 


\section{2 结论}

本文介绍了一种在哌啶溶剂中, 以廉价 $\mathrm{Cu}(\mathrm{OAc})_{2}$ 为催化剂, 利用乙炔澳与丙炔醇为反应底物通过偶联异构化反应专一的生成 $(E)$-1,5-二取代-戊-2-烯-4-炔-1酮的方法. 该反应具有立体专一、条件温和、产率较高、 操作简单和目标产物容易分离的优点, 为合成反式 1,5二取代戊炔烯酮类化合物提供了一条新的途径.

\section{3 实验部分}

\section{1 仪器与试剂}

所用试剂均为市售的分析纯, 除特别说明外, 未经 进一步处理. ${ }^{1} \mathrm{H}$ NMR 和 ${ }^{13} \mathrm{C}$ NMR 用 Varian INOVA-500 测定, 以 $\mathrm{TMS}$ 为内标, $\mathrm{CDCl}_{3}$ 作溶剂; 高分辨质谱使用 Bruker micrOTOF II 仪器测定; 熔点用 X-4 数字显示显 微熔点测定仪; 单晶仪 Oxford Diffraction Gemini R Ultra diffractometer; 柱层析使用青岛海洋化工厂生产的硅 胶 $\mathrm{G}$ (颗粒度 $10 \sim 40 \mu \mathrm{m}$ ).

\section{2 实验方法}

以合成 $(E)$-5-(4-氯苯基)-1-苯基-成-2-烯-4-炔-1-酮 (3a) 为例. 在 $\mathrm{N}_{2}$ 气氛下, 将哌啶 $(2.0 \mathrm{~mL})$ 加入到装有炔 溴 1a $(0.107 \mathrm{~g}, 0.5 \mathrm{mmol})$ 和炔醇 $2 \mathrm{a}(0.086 \mathrm{~g}, 0.65 \mathrm{mmol})$ 圆底烧瓶中, 冰浴条件下搅拌 $10 \mathrm{~min}$ 后, 再加入醋酸铜 $(0.010 \mathrm{~g}, 0.05 \mathrm{mmol})$, 反应搅拌 $1 \mathrm{~h}$ 后升温至室温, 在室 温下摚拌过夜 $(12 \mathrm{~h})$. TLC 监测反应结束后, 将混合物倒 入饱和氯化铵水溶液中, 分离有机层, 水层用二氯甲烷 萃取 $(25 \mathrm{~mL} \times 3)$. 将有机层和萃取液合并, 用无水硫酸 镁干燥, 过滤, 将滤液浓缩, 上硅胶柱层析分离, 用石 油醚/乙酸乙酯 $(V: V=90 ： 1)$, 得到 3a $(0.109 \mathrm{~g}, 82 \%)$.

(E)-5-(4-氯苯基)-1-苯基-戊-2-烯-4-炔-1-酮(3a)：淡 黄色固体. m.p. $110 \sim 111{ }^{\circ} \mathrm{C} ;{ }^{1} \mathrm{H}$ NMR $(500 \mathrm{MHz}$, $\left.\mathrm{CDCl}_{3}\right) \delta: 7.10(\mathrm{~d}, J=15.5 \mathrm{~Hz}, 1 \mathrm{H}), 7.33(\mathrm{~d}, J=8.5 \mathrm{~Hz}$, $2 \mathrm{H}), 7.42 \sim 7.45(\mathrm{~m}, 3 \mathrm{H}), 7.49(\mathrm{t}, J=8.0 \mathrm{~Hz}, 2 \mathrm{H}), 7.58(\mathrm{t}$, $J=7.5 \mathrm{~Hz}, 1 \mathrm{H}), 7.98(\mathrm{~d}, J=7.5 \mathrm{~Hz}, 2 \mathrm{H}) ;{ }^{13} \mathrm{C} \mathrm{NMR}$ $\left(\mathrm{CDCl}_{3}, 125 \mathrm{MHz}\right) \delta$ : 88.6, 97.8, 120.7, 124.6, 128.5, $128.7,128.8,133.1,133.2,133.3,135.5,137.1,188.6$; HRMS (ESI) calcd for $\mathrm{C}_{17} \mathrm{H}_{12} \mathrm{ClO}[\mathrm{M}+\mathrm{H}]^{+}$267.0577, found 267.0589 .

(E)-1,5-二苯基-戊-2-烯-4-炔-1-酮(3b)：淡黄色固 体. m.p. 99 100 ${ }^{\circ} \mathrm{C} ;{ }^{1} \mathrm{H}$ NMR $\left(500 \mathrm{MHz}, \mathrm{CDCl}_{3}\right) \delta: 7.14$ (d, $J=15.5 \mathrm{~Hz}, 1 \mathrm{H}), 7.35 \sim 7.41$ (m, 3H), 7.45 (d, $J=15.5$ $\mathrm{Hz}, 1 \mathrm{H}), 7.49 \sim 7.54$ (m, 4H), 7.60 (t, $J=7.5 \mathrm{~Hz}, 1 \mathrm{H}), 8.00$ $(\mathrm{d}, J=7.5 \mathrm{~Hz}, 2 \mathrm{H}) ;{ }^{13} \mathrm{C} \mathrm{NMR}\left(\mathrm{CDCl}_{3}, 125 \mathrm{MHz}\right) \delta: 87.7$, $99.3,122.2,125.1,128.49,128.54,128.7,129.4,132.0$, 133.0, 133.2, 137.2, 188.8; HRMS (ESI) calcd for $\mathrm{C}_{17} \mathrm{H}_{13} \mathrm{O}$
$[\mathrm{M}+\mathrm{H}]^{+}$233.0966, found 233.0968.

(E)-5-(4-甲氧基苯基)-1-苯基-戊-2-烯-4-炔-1-酮 (3c): 淡黄色固体. m.p. 96 97 ${ }^{\circ} \mathrm{C} ;{ }^{1} \mathrm{H}$ NMR $(500 \mathrm{MHz}$, $\left.\mathrm{CDCl}_{3}\right) \delta: 3.84(\mathrm{~s}, 3 \mathrm{H}), 6.89(\mathrm{~d}, J=8.5 \mathrm{~Hz}, 2 \mathrm{H}), 7.14(\mathrm{~d}$, $J=15.5 \mathrm{~Hz}, 1 \mathrm{H}), 7.40(\mathrm{~d}, J=15.5 \mathrm{~Hz}, 1 \mathrm{H}), 7.44 \sim 7.52(\mathrm{~m}$, $4 \mathrm{H}), 7.59(\mathrm{t}, J=7.5 \mathrm{~Hz}, 1 \mathrm{H}), 8.00(\mathrm{~d}, J=7.5 \mathrm{~Hz}, 2 \mathrm{H}) ;{ }^{13} \mathrm{C}$ NMR $\left(\mathrm{CDCl}_{3}, 125 \mathrm{MHz}\right) \delta: 55.3,87.1,100.1,114.1$, $114.2,125.5,128.5,128.6,132.0,133.0,133.7,137.3$, 160.5, 188.9; HRMS (ESI) calcd for $\mathrm{C}_{18} \mathrm{H}_{15} \mathrm{O}_{2}[\mathrm{M}+\mathrm{H}]^{+}$ 263.1072 , found 263.1073 .

(E)-5-(3-甲氧基苯基)-1-苯基-戊-2-烯-4-炔-1-酮 (3d): 淡黄色液体; ${ }^{1} \mathrm{H}$ NMR $\left(500 \mathrm{MHz}, \mathrm{CDCl}_{3}\right) \delta: 3.82$ (s, $3 \mathrm{H}), 6.95(\mathrm{dd}, J=2.5,8.5 \mathrm{~Hz}, 1 \mathrm{H}), 7.03 \sim 7.06(\mathrm{~m}, 1 \mathrm{H})$, $7.10 \sim 7.16(\mathrm{~m}, 2 \mathrm{H}), 7.25 \sim 7.29(\mathrm{~m}, 1 \mathrm{H}), 7.45(\mathrm{~d}, J=15.5$ $\mathrm{Hz}, 1 \mathrm{H}), 7.48 \sim 7.53(\mathrm{t}, J=8.0 \mathrm{~Hz}, 2 \mathrm{H}), 7.60$ (t, $J=7.5 \mathrm{~Hz}$, $1 \mathrm{H}), 8.00(\mathrm{~d}, J=8.0 \mathrm{~Hz}, 2 \mathrm{H}) ;{ }^{13} \mathrm{C} \mathrm{NMR}\left(\mathrm{CDCl}_{3}, 125 \mathrm{MHz}\right)$ $\delta: 55.3,87.5,99.2,116.1,116.6,123.1,124.6,125.0$, $128.5,128.7,129.6,133.1,133.2,137.1,159.3,188.8$; HRMS (ESI) calcd for $\mathrm{C}_{18} \mathrm{H}_{15} \mathrm{O}_{2}[\mathrm{M}+\mathrm{H}]^{+}$263.1072, found 263.1066 .

(E)-5-(4-氟苯基)-1-苯基-戊-2-烯-4-炔-1-酮(3e)：淡 黄色固体. m.p. $76 \sim 77{ }^{\circ} \mathrm{C} ;{ }^{1} \mathrm{H}$ NMR $\left(500 \mathrm{MHz}, \mathrm{CDCl}_{3}\right)$ $\delta: 7.05 \sim 7.13(\mathrm{~m}, 3 \mathrm{H}), 7.44(\mathrm{~d}, J=15.5 \mathrm{~Hz}, 1 \mathrm{H}), 7.49 \sim$ $7.52(\mathrm{~m}, 4 \mathrm{H}), 7.60(\mathrm{t}, J=7.5 \mathrm{~Hz}, 1 \mathrm{H}), 8.00(\mathrm{~d}, J=7.5 \mathrm{~Hz}$, $2 \mathrm{H}) ;{ }^{13} \mathrm{C}$ NMR $\left(\mathrm{CDCl}_{3}, 125 \mathrm{MHz}\right) \delta: 87.5,98.2,115.8(\mathrm{~d}$, $J=22.0 \mathrm{~Hz}), 118.3(\mathrm{~d}, J=3.5 \mathrm{~Hz}), 124.9,128.5,128.7$, $133.0,133.3,134.0$ (d, $J=8.5 \mathrm{~Hz}), 137.1,162.1$ (d, $J=$ $252.0 \mathrm{~Hz}$ ), 188.8; HRMS (ESI) calcd for $\mathrm{C}_{17} \mathrm{H}_{12} \mathrm{FO}[\mathrm{M}+$ $\mathrm{H}]^{+}$251.0872, found 251.0849 .

(E)-5-(萗-2-基)-1-苯基-戊-2-烯-4-炔-1-酮(3f)：淡黄 色固体. m.p. 79 $80{ }^{\circ} \mathrm{C} ;{ }^{1} \mathrm{H}$ NMR $\left(500 \mathrm{MHz}, \mathrm{CDCl}_{3}\right) \delta$ : $7.20(\mathrm{~d}, J=15.5 \mathrm{~Hz}, 1 \mathrm{H}), 7.47 \sim 7.55(\mathrm{~m}, 6 \mathrm{H}), 7.60$ (t, $J=$ $7.5 \mathrm{~Hz}, 1 \mathrm{H}), 7.82 \sim 7.84(\mathrm{~m}, 3 \mathrm{H}), 8.01(\mathrm{~d}, J=7.5 \mathrm{~Hz}, 2 \mathrm{H})$, $8.07(\mathrm{~s}, 1 \mathrm{H}) ;{ }^{13} \mathrm{C}$ NMR $\left(\mathrm{CDCl}_{3}, 125 \mathrm{MHz}\right) \delta: 88.2,99.8$, $119.5,125.2,126.8,127.3,127.8,128.0,128.20,128.24$, $128.6,128.7,132.5,132.8,133.0,133.2,133.3,137.2$, 188.8; HRMS (ESI) calcd for $\mathrm{C}_{21} \mathrm{H}_{15} \mathrm{O}[\mathrm{M}+\mathrm{H}]^{+}$ 283.1123, found 283.1125.

(E)-1-苯基-5-(噻吩-2-基)-戊-2-烯-4-炔-1-酮 (3g): 红褐色油状液体. ${ }^{1} \mathrm{H}$ NMR $\left(500 \mathrm{MHz}, \mathrm{CDCl}_{3}\right) \delta: 7.02 \sim$ $7.05(\mathrm{~m}, 1 \mathrm{H}), 7.13(\mathrm{~d}, J=15.5 \mathrm{~Hz}, 1 \mathrm{H}), 7.33 \sim 7.37(\mathrm{~m}$, $1 \mathrm{H}), 7.38 \sim 7.45(\mathrm{~m}, 2 \mathrm{H}), 7.50(\mathrm{t}, J=7.5 \mathrm{~Hz}, 2 \mathrm{H}), 7.60(\mathrm{t}$, $J=7.5 \mathrm{~Hz}, 1 \mathrm{H}), 8.00(\mathrm{~d}, J=7.0 \mathrm{~Hz}, 2 \mathrm{H}) ;{ }^{13} \mathrm{C} \mathrm{NMR}$ $\left(\mathrm{CDCl}_{3}, 125 \mathrm{MHz}\right) \delta: 92.2,92.8,122.2,124.6,127.5$, $128.5,128.7,129.4,132.3,133.2,133.8,137.1,188.6$; 
HRMS (ESI) calcd for $\mathrm{C}_{15} \mathrm{H}_{11} \mathrm{OS}[\mathrm{M}+\mathrm{H}]^{+}$239.0531, found 239.0531 .

(E)-6-(2-溴苯氧基)-1-苯基-己-2-烯-4-炔-1-酮(3h): 白色固体. m.p. 88 89 ${ }^{\circ} \mathrm{C}$; ${ }^{1} \mathrm{H}$ NMR (500 $\mathrm{MHz}, \mathrm{CDCl}_{3}$ ) $\delta: 5.00(\mathrm{~s}, 2 \mathrm{H}), 6.88 \sim 6.93(\mathrm{~m}, 2 \mathrm{H}), 7.07(\mathrm{~d}, J=8.0 \mathrm{~Hz}$, 1H), $7.26 \sim 7.32(\mathrm{~m}, 1 \mathrm{H}), 7.36(\mathrm{~d}, J=15.5 \mathrm{~Hz}, 1 \mathrm{H}), 7.49$ $(\mathrm{t}, J=7.5 \mathrm{~Hz}, 2 \mathrm{H}), 7.56 \sim 7.62(\mathrm{~m}, 2 \mathrm{H}), 7.94(\mathrm{~d}, J=7.5$ $\mathrm{Hz}, 2 \mathrm{H}) ;{ }^{13} \mathrm{C} \mathrm{NMR}\left(\mathrm{CDCl}_{3}, 125 \mathrm{MHz}\right) \delta: 57.5,85.6,92.9$, $112.4,114.1,123.0,123.7,128.4,128.6,128.8,133.4$, 133.6, 134.3, 136.8, 153.9, 188.6; HRMS (ESI) calcd for $\mathrm{C}_{18} \mathrm{H}_{14} \mathrm{O}_{2}[\mathrm{M}+\mathrm{H}]^{+}$341.0177, found 341.0169.

(E)-6-氧代-6-苯基-己-4-烯-2-炔-1-基苯甲酸乙酯 (3i): 淡黄色固体. m.p. 79 80 ${ }^{\circ} \mathrm{C}$; ${ }^{1} \mathrm{H}-\mathrm{NMR}(500 \mathrm{MHz}$, $\left.\mathrm{CDCl}_{3}\right) \delta: 5.13(\mathrm{~s}, 2 \mathrm{H}), 6.89 \sim 6.94(\mathrm{~m}, 1 \mathrm{H}), 7.40(\mathrm{~d}, J=$ $15.5 \mathrm{~Hz}, 1 \mathrm{H}), 7.44 \sim 7.51(\mathrm{~m}, 4 \mathrm{H}), 7.55 \sim 7.62(\mathrm{~m}, 2 \mathrm{H})$, $7.95(\mathrm{~d}, J=7.5 \mathrm{~Hz}, 2 \mathrm{H}), 8.09(\mathrm{~d}, J=7.5 \mathrm{~Hz}, 2 \mathrm{H}) ;{ }^{13} \mathrm{C}$ $\mathrm{NMR}\left(\mathrm{CDCl}_{3}, 125 \mathrm{MHz}\right) \delta: 52.9,84.5,92.7,123.8,128.4$, $128.5,128.7$, 129.2, 129.8, 133.3, 133.4, 134.3, 136.8, 165.7, 188.5; HRMS (ESI) calcd for $\mathrm{C}_{19} \mathrm{H}_{15} \mathrm{O}_{3}[\mathrm{M}+\mathrm{H}]^{+}$ 291.1021, found 291.1019.

(E)-1-苯基壬-2-烯-4-炔-1-酮(3j): 淡黄色液体; ${ }^{1} \mathrm{H}$ NMR (500 MHz, $\left.\mathrm{CDCl}_{3}\right) \delta: 0.94(\mathrm{t}, J=7.5 \mathrm{~Hz}, 3 \mathrm{H})$, $1.42 \sim 1.49(\mathrm{~m}, 2 \mathrm{H}), 1.54 \sim 1.60(\mathrm{~m}, 2 \mathrm{H}), 2.40 \sim 2.45(\mathrm{~m}$, 2H), $6.90(\mathrm{dt}, J=2.4,15.5 \mathrm{~Hz}, 1 \mathrm{H}), 7.27$ (d, $J=15.5 \mathrm{~Hz}$, $1 \mathrm{H}), 7.45 \sim 7.50(\mathrm{~m}, 2 \mathrm{H}), 7.55 \sim 7.60(\mathrm{~m}, 1 \mathrm{H}), 7.93 \sim 7.97$ $(\mathrm{m}, 2 \mathrm{H}) ;{ }^{13} \mathrm{C} \mathrm{NMR}\left(\mathrm{CDCl}_{3}, 125 \mathrm{MHz}\right) \delta: 13.6,19.6,22.0$, 30.4, 79.2, 102.1, 126.3, 128.5, 128.6, 132.5, 133.0, 137.3, 189.2; HRMS (ESI) calcd for $\mathrm{C}_{15} \mathrm{H}_{17} \mathrm{O}[\mathrm{M}+\mathrm{H}]^{+}$ 213.1279, found 213.1270.

(E)-5-(4-氯苯基)-1-对甲苯基-戍-2-烯-4-炔-1-酮 (3k): 淡黄色固体. m.p. $121 \sim 122{ }^{\circ} \mathrm{C} ;{ }^{1} \mathrm{H}$ NMR (500 $\left.\mathrm{MHz} \mathrm{CDCl}_{3}\right) \delta: 2.43(\mathrm{~s}, 3 \mathrm{H}), 7.09(\mathrm{~d}, J=15.5 \mathrm{~Hz}, 1 \mathrm{H})$, $7.29(\mathrm{~d}, J=8.0 \mathrm{~Hz}, 2 \mathrm{H}), 7.33 \sim 7.34(\mathrm{~m}, 2 \mathrm{H}), 7.42 \sim 7.44$ (m, 3H), $7.90(\mathrm{~d}, J=8.0 \mathrm{~Hz}, 2 \mathrm{H}) ;{ }^{13} \mathrm{C} \mathrm{NMR}\left(\mathrm{CDCl}_{3}, 125\right.$ $\mathrm{MHz}) \delta: 21.7,88.7,97.5,120.8,124.1,128.7,128.8$, 129.4, 133.1, 133.4, 134.6, 135.4, 144.2, 188.1; HRMS (ESI) calcd for $\mathrm{C}_{18} \mathrm{H}_{14} \mathrm{ClO}[\mathrm{M}+\mathrm{H}]^{+} 281.0733$, found 281.0742 .

(E)-5-(4-氯苯基)-1-(3-三氟甲基苯基)-戊-2-烯-4-炔 -1-酮(3I): 淡黄色固体. m.p. 76 77 ${ }^{\circ} \mathrm{C} ;{ }^{1} \mathrm{H}$ NMR (500 $\left.\mathrm{MHz} \mathrm{CDCl}_{3}\right) \delta: 7.16(\mathrm{~d}, J=15.5 \mathrm{~Hz}, 1 \mathrm{H}), 7.36$ (d, $J=8.5$ $\mathrm{Hz}, 2 \mathrm{H}), 7.42 \sim 7.47(\mathrm{~m}, 3 \mathrm{H}), 7.66(\mathrm{t}, J=8.0 \mathrm{~Hz}, 1 \mathrm{H}), 7.86$ $(\mathrm{d}, J=7.5 \mathrm{~Hz}, 1 \mathrm{H}), 8.17(\mathrm{~d}, J=8.0 \mathrm{~Hz}, 1 \mathrm{H}), 8.24(\mathrm{~s}, 1 \mathrm{H})$; ${ }^{13} \mathrm{C} \mathrm{NMR}\left(\mathrm{CDCl}_{3}, 125 \mathrm{MHz}\right) \delta: 88.4,99.0,120.5,122.5$ (q, $J=272.0 \mathrm{~Hz}), 125.3$ (q, $J=3.5 \mathrm{~Hz}), 126.0,128.9,129.4$,
129.6 (q, $J=3.5 \mathrm{~Hz}), 131.2$ (q, $J=3.5 \mathrm{~Hz}), 131.6,132.3$, 133.3, 135.8, 137.7, 187.3; HRMS (ESI) calcd for $\mathrm{C}_{18} \mathrm{H}_{11} \mathrm{ClF}_{3} \mathrm{O}[\mathrm{M}+\mathrm{H}]^{+}$335.0451, found 335.0437.

(E)-5-(4-氯苯基)-1-(2,4-二氯苯基)-成-2-烯-4-炔-1酮(3m): 淡黄色固体. m.p. 89 90 ${ }^{\circ} \mathrm{C} ;{ }^{1} \mathrm{H}$ NMR (500 $\left.\mathrm{MHz}, \mathrm{CDCl}_{3}\right) \delta: 6.85(\mathrm{~d}, J=16.0 \mathrm{~Hz}, 1 \mathrm{H}), 7.01(\mathrm{~d}, J=$ $16.0 \mathrm{~Hz}, 1 \mathrm{H}), 7.32 \sim 7.35(\mathrm{~m}, 3 \mathrm{H}), 7.41 \sim 7.46(\mathrm{~m}, 4 \mathrm{H})$; ${ }^{13} \mathrm{C} \mathrm{NMR}\left(\mathrm{CDCl}_{3}, 125 \mathrm{MHz}\right) \delta: 88.0,100.0,120.4,126.0$, $127.4,128.9,130.3,130.6,132.6,133.2,135.8,136.4$, 136.6, 137.5, 190.8; HRMS (ESI) calcd for $\mathrm{C}_{17} \mathrm{H}_{10} \mathrm{Cl}_{3} \mathrm{O}$ $[\mathrm{M}+\mathrm{H}]^{+}$334.9797, found 334.9797 .

(1 $E, 4 E)$-7-(4-氯苯基)-1-苯基-庚-1,4-二烯-6-炔-3-酮 (3n): 淡黄色固体. m.p. $119 \sim 120{ }^{\circ} \mathrm{C} ;{ }^{1} \mathrm{H}$ NMR (500 $\left.\mathrm{MHz}, \mathrm{CDCl}_{3}\right) \delta: 6.93(\mathrm{~d}, J=16.0 \mathrm{~Hz}, 1 \mathrm{H}), 6.96 \sim 7.05(\mathrm{~m}$, $2 \mathrm{H}), 7.33(\mathrm{~d}, J=7.5 \mathrm{~Hz}, 2 \mathrm{H}), 7.42 \sim 7.44(\mathrm{~m}, 5 \mathrm{H}), 7.55 \sim$ $7.62(\mathrm{~m}, 2 \mathrm{H}), 7.71(\mathrm{~d}, J=16.0 \mathrm{~Hz}, 1 \mathrm{H}) ;{ }^{13} \mathrm{C} \mathrm{NMR}\left(\mathrm{CDCl}_{3}\right.$, $125 \mathrm{MHz}) \delta: 88.4,97.9,120.7,123.3,125.3,128.4,128.9$, 129.0, 133.2, 134.2, 134.4, 135.5, 136.1, 144.2, 187.5; HRMS (ESI) calcd for $\mathrm{C}_{19} \mathrm{H}_{14} \mathrm{ClO}[\mathrm{M}+\mathrm{H}]^{+}$293.0733, found 293.0719 .

(E)-1-(苯并 $[d][1,3]$ 二氧杂环戊烯-5-基)-5-(4-氯苯 基)-戊-2-烯-4-炔-1-酮 (3o): 白色固体. m.p. 118 $119{ }^{\circ} \mathrm{C} ;{ }^{1} \mathrm{H}$ NMR $\left(500 \mathrm{MHz}, \mathrm{CDCl}_{3}\right) \delta: 6.07$ (s, 2H), 6.88 $(\mathrm{d}, J=8.0 \mathrm{~Hz}, 1 \mathrm{H}), 7.75(\mathrm{~d}, J=15.5 \mathrm{~Hz}, 1 \mathrm{H}), 7.34(\mathrm{~d}, J=$ $8.5 \mathrm{~Hz}, 2 \mathrm{H}), 7.38 \sim 7.44(\mathrm{~m}, 3 \mathrm{H}), 7.49$ (d, $J=1.5 \mathrm{~Hz}, 1 \mathrm{H})$, $7.60(\mathrm{dd}, J=1.5,8.0 \mathrm{~Hz}, 1 \mathrm{H}) ;{ }^{13} \mathrm{C} \mathrm{NMR}\left(\mathrm{CDCl}_{3}, 125\right.$ $\mathrm{MHz}) \delta: 88.6,97.4,101.9,107.9,108.2,120.7,124.0$, $125.0,128.8,131.9,133.1,133.3,135.4,148.4,152.1$, 186.4; HRMS (ESI) calcd for $\mathrm{C}_{18} \mathrm{H}_{12} \mathrm{ClO}_{3}[\mathrm{M}+\mathrm{H}]^{+}$ 311.0475 , found 311.0477 .

(E)-5-(4-氯苯基)-1-(菜-2-基)-戍-2-烯-4-炔-1-酮 (3p): 淡黄色固体. m.p. $118 \sim 119{ }^{\circ} \mathrm{C} ;{ }^{1} \mathrm{H}$ NMR (500 $\left.\mathrm{MHz} \mathrm{CDCl}_{3}\right) \delta: 7.17(\mathrm{~d}, J=15.5 \mathrm{~Hz}, 1 \mathrm{H}), 7.36(\mathrm{~d}, J=8.5$ $\mathrm{Hz}, 2 \mathrm{H}), 7.47(\mathrm{~d}, J=8.5 \mathrm{~Hz}, 2 \mathrm{H}), 7.56 \sim 7.64(\mathrm{~m}, 3 \mathrm{H})$, 7.90 (d, $J=8.0 \mathrm{~Hz}, 1 \mathrm{H}), 7.94$ (d, $J=8.0 \mathrm{~Hz}, 1 \mathrm{H}), 7.99$ (d, $J=8.0 \mathrm{~Hz}, 1 \mathrm{H}), 8.07$ (dd, $J=1.5,8.5 \mathrm{~Hz}, 1 \mathrm{H}), 8.51$ (s, $1 \mathrm{H}) ;{ }^{13} \mathrm{C} \mathrm{NMR}\left(\mathrm{CDCl}_{3}, 125 \mathrm{MHz}\right) \delta: 88.7,97.9,120.7$, $124.2,124.6,126.9,127.8,128.7,128.8,128.9,129.6$, $130.3,132.5,133.2,133.3,134.5,135.6,135.7,188.4$; HRMS (ESI) calcd for $\mathrm{C}_{21} \mathrm{H}_{14} \mathrm{ClO}[\mathrm{M}+\mathrm{H}]^{+}$317.0733, found 317.0738 .

(E)-5-(4-氯苯基)-1-(吡啶-3-基)-戍-2-烯-4-炔-1-酮 (3q): 淡黄色固体. m.p. 98 99 ${ }^{\circ} \mathrm{C} ;{ }^{1} \mathrm{H}$ NMR (500 MHz, $\left.\mathrm{CDCl}_{3}\right) \delta: 7.17(\mathrm{~d}, J=15.5 \mathrm{~Hz}, 1 \mathrm{H}), 7.36(\mathrm{~d}, J=8.5 \mathrm{~Hz}$, $2 \mathrm{H}), 7.41(\mathrm{~d}, J=15.5 \mathrm{~Hz}, 1 \mathrm{H}), 7.46 \sim 7.48(\mathrm{~m}, 3 \mathrm{H}), 8.26 \sim$ 
$8.30(\mathrm{~m}, 1 \mathrm{H}), 8.80 \sim 8.84(\mathrm{~m}, 1 \mathrm{H}), 9.21(\mathrm{~d}, J=1.5 \mathrm{~Hz}$, $1 \mathrm{H}) ;{ }^{13} \mathrm{C} \mathrm{NMR}\left(\mathrm{CDCl}_{3}, 125 \mathrm{MHz}\right) \delta: 88.3,99.2,120.4$, $123.7,125.9,128.9,132.3,132.5,133.3,135.8,135.9$, 149.8, 153.6, 187.4; HRMS (ESI) calcd for $\mathrm{C}_{16} \mathrm{H}_{11} \mathrm{CINO}$ $[\mathrm{M}+\mathrm{H}]^{+}$268.0529, found 268.0546 .

(E)-5-(4-氯苯基)-1-(噻吩-2-基)-戊-2-烯-4-炔-1-酮 (3r): 白色固体. m.p. 117 $118{ }^{\circ} \mathrm{C} ;{ }^{1} \mathrm{H}$ NMR $(500 \mathrm{MHz}$, $\left.\mathrm{CDCl}_{3}\right) \delta: 7.12(\mathrm{~d}, J=15.5 \mathrm{~Hz}, 1 \mathrm{H}), 7.18(\mathrm{t}, J=4.0 \mathrm{~Hz}$, $1 \mathrm{H}), 7.29 \sim 7.35(\mathrm{~m}, 3 \mathrm{H}), 7.44(\mathrm{~d}, J=8.5 \mathrm{~Hz}, 2 \mathrm{H}), 7.71$ (d, $J=5.0 \mathrm{~Hz}, 1 \mathrm{H}), 7.81(\mathrm{~d}, J=3.5 \mathrm{~Hz}, 1 \mathrm{H}) ;{ }^{13} \mathrm{C} \mathrm{NMR}$ $\left(\mathrm{CDCl}_{3}, 125 \mathrm{MHz}\right) \delta: 88.4,98.0,120.6,124.0,128.4$, $128.9,132.4,133.1,133.2,134.7,135.6,144.6,180.6$; HRMS (ESI) calcd for $\mathrm{C}_{15} \mathrm{H}_{10} \mathrm{ClOS}[\mathrm{M}+\mathrm{H}]^{+}$273.0141, found 273.0144 .

5-(4-氯苯基)-1-环己基-戊-2,4-二炔-1-醇(3s): 白色 固体. m.p. $77 \sim 78{ }^{\circ} \mathrm{C} ;{ }^{1} \mathrm{H}$ NMR $\left(500 \mathrm{MHz}, \mathrm{CDCl}_{3}\right) \delta$ : $1.06 \sim 1.24(\mathrm{~m}, 5 \mathrm{H}), 1.59 \sim 1.66(\mathrm{~m}, 1 \mathrm{H}), 1.66 \sim 1.72(\mathrm{~m}$, $1 \mathrm{H}), 1.75 \sim 1.82(\mathrm{~m}, 2 \mathrm{H}), 1.84 \sim 1.92(\mathrm{~m}, 3 \mathrm{H}), 4.30(\mathrm{~d}, J=$ $6.0 \mathrm{~Hz}, 1 \mathrm{H}), 7.30(\mathrm{~d}, J=8.5 \mathrm{~Hz}, 2 \mathrm{H}), 7.41$ (d, $J=8.5 \mathrm{~Hz}$, $2 \mathrm{H}) ;{ }^{13} \mathrm{C} \mathrm{NMR}\left(\mathrm{CDCl}_{3}, 125 \mathrm{MHz}\right) \delta: 25.7,25.8,26.3,28.1$, 28.5, 44.2, 67.8, 70.0, 74.2, 77.1, 83.2, 120.0, 128.8, 133.8, 135.4; HRMS (ESI) calcd for $\mathrm{C}_{17} \mathrm{H}_{17} \mathrm{ClO}[\mathrm{M}+\mathrm{H}]^{+}$ 272.0968, found 272.0979 .

5-(4-氯苯基)-3-(哌啶-1-基)-1-对甲苯基-戊-4-炔-1酮 $(\mathbf{F})$ : 淡黄色固体. m.p. 97 98 ${ }^{\circ} \mathrm{C} ;{ }^{1} \mathrm{H}$ NMR (500 $\left.\mathrm{MHz}, \mathrm{CDCl}_{3}\right) \delta: 1.39 \sim 1.48(\mathrm{~m}, 2 \mathrm{H}), 1.49 \sim 1.68(\mathrm{~m}, 4 \mathrm{H})$, $2.41(\mathrm{~s}, 3 \mathrm{H}), 2.51 \sim 2.60(\mathrm{~m}, 2 \mathrm{H}), 2.65 \sim 2.73(\mathrm{~m}, 2 \mathrm{H})$, $3.29 \sim 3.34(\mathrm{~m}, 1 \mathrm{H}), 3.40 \sim 3.48(\mathrm{~m}, 1 \mathrm{H}), 4.29(\mathrm{dd}, J=5.0$, $8.5 \mathrm{~Hz}, 1 \mathrm{H}), 7.22 \sim 7.29(\mathrm{~m}, 6 \mathrm{H}), 7.88$ (d, $J=8 \mathrm{~Hz}, 2 \mathrm{H})$; ${ }^{13} \mathrm{C} \mathrm{NMR}\left(\mathrm{CDCl}_{3}, 125 \mathrm{MHz}\right) \delta: 21.6,24.3,26.0,42.5$, 50.9, 54.1, 84.8, 88.0, 121.6, 128.2, 128.4, 129.2, 132.9, 133.8, 134.5, 143.9, 196.8; HRMS (ESI) calcd for $\mathrm{C}_{23} \mathrm{H}_{25} \mathrm{CINO}[\mathrm{M}+\mathrm{H}]^{+}$366.1625, found 366.1607 .

辅助材料(Supporting Information) 目标化合物 3a $3 \mathbf{s}$ 的 ${ }^{1} \mathrm{H}$ NMR, ${ }^{13} \mathrm{C}$ NMR 谱图和 $3 \mathbf{r}$ 的晶体结构数据和图. 这些材料可以免费从本刊网站(http://sioc-journal.cn/)上 下载.

\section{References}

[1] (a) Miki, K.; Nishino, F.; Ohe, K.; Uemura, S. J. Am. Chem. Soc. 2002, 124, 5260 .

(b) Miki, K.; Yokoi, T.; Nishino, F.; Kato, Y.; Washitake, Y.; Ohe, K.; Uemura, S. J. Org. Chem. 2004, 69, 1557.

(c) Du, X.; Chen, H.; Chen, Y.; Chen, J.; Liu, Y. Synlett 2011, 1010 .

(d) Hirata, Y.; Tanaka, M.; Yada, A.; Nakao, Y.; Hiyama, T. Tet- rahedron 2009, 65, 5037.

[2] (a) Sukhova, L. N.; Ostroumov, I. G.; Belskii, V. K.; Gali-shev, V. A.; Maretina, I. A. Zh. Org. Khim. 1994, 30, 46.

(b) Waldo, J. P.; Mehta, S.; Larock, R.C. J. Org. Chem. 2008, 73, 6666.

[3] (a) Iwasawa, N.; Shido, M.; Maeyama, K.; Kusama, H. J. Am. Chem. Soc. 2000, 122, 10226.

(b) Ohe, K.; Miki, K.; Yokoi, T.; Nishino, F.; Uemura, S. Organometallics 2000, 19, 5525 .

(c) Iwasawa, N.; Shido, M.; Kusama, H. J. Am. Chem. Soc. 2001, 123, 5814.

[4] Rosiak, A.; Christoffers, J. Tetrahedron Lett. 2006, 47, 5095.

[5] (a) Oliva, C. G.; Silva, A. M. S.; Paz, F. A.;A.; Cavaleiro, J. A. S. Synlett 2010, 1123.

(b) Toshima, H.; Aramaki, H.; Ichihara, A. Tetrahedron Lett. 1999, $40,3587$.

[6] Chen, J. J.; Fan, G. Q.; Liu, Y. H. Org. Biomol. Chem. 2010, 8, 4806.

[7] (a) Hadi, V.; Yoo, K. S.; Jeong, M.; Jung, K. W. Tetrahedron Lett. 2009, 50, 2370.

(b) Martins, M. A. P.; Rossatto, M.; Rosa, F. A.; Machado, P.; Zanatta, N.; Bonacorso, H. G. ARKIVOC 2007, Part (i), 205.

[8] Golovanov, A. A.; Latypova, D. R.; Bekin, V. V.; Pisareva, V. S.; Vologzhanina, A. V.; Dokichev, V. A. Russian J. Org. Chem. 2013, 49, 1264.

[9] Nishimura, T.; Araki, H.; Maeda, Y.; Uemura, S. Org. Lett. 2003, $5,2997$.

[10] Xie, Y. J.; Yu, M.; Zhang, Y. H. Synthesis 2011, 2803.

[11] (a) Boukouvalas, J.; Sebastien, C.; Ndzi, B. Tetrahedron Lett. 2007, $48,105$.

(b) Fu, X.; Zhang, S.; Yin, J.; Schumacher, D. Tetrahedron Lett. 2002, 43, 6673.

(c) Fiandanese, V.; Bottalico, D.; Marchese, G.; Punzi, A. Tetrahedron 2006, 62, 5126.

(d) Emna, Z.; Mohamed Moncef, E. G. J. Chem. Res. 2010, $34,5$.

(e) Daia, D. E.; Gabbutt, C. D.; Heron, B. M.; Hepworth, J. D.; Hursthouse. M. B.; Abdul Malik, K. M. Tetrahedron Lett. 2003, 44, 1461.

[12] Dong, L. J.; Fan, T. T.; Wang, C.; Sun, J. Org. Lett. 2013, 15, 204.

[13] (a) Cera, G.; Piscitelli, S.; Chiarucci, M.; Fabrizi, G.; Goggiamani, A.; Ramón, R. S.; Nolan, S. P.; Bandini, M. Angew. Chem., Int. Ed. 2012, 51, 9891.

(b) Chinkov, N.; Warm, A.; Carreira, E. M. Angew. Chem., Int. Ed. 2011, 50, 2957.

(c) Miura, T.; Funakoshi, Y.; Morimoto, M.; Biyajima, T.; Murakami, M. J. Am. Chem. Soc. 2012, 134, 17440.

(d) Ye, L.; He, W.; Zhang, L. J. Am. Chem. Soc. 2010, 132, 8550.

[14] (a) Yoshizawa, K.; Shioiri, T. Tetrahedron 2007, 63, 6259.

(b) Braun, R. U.; Ansorge, M.; Müller, T. J. J. Chem.-Eur. J. 2006, 12, 9081 .

(c) Schramm, O. G.; Müller, T. J. J. Adv. Synth. Catal. 2006, 348, 2565 .

(d) Ishikawa, T.; Mizuta, T.; Hagiwara, K.; Aikawa, T.; Kudo, T.; Saito, S. J. Org. Chem. 2003, 68, 3702.

(e) Cho, C. S.; Lee, N. Y.; Kim, T.-J.; Shim, S. C. J. Heterocycl. Chem. 2004, 41, 409.

(f) Sonye, J. P.; Koide, K. Org. Lett. 2006, 8, 199.

[15] (a) Liu, Z.; Liu, J.; Zhang, L.; Liao, P.; Song, J.; Bi, X. Angew. Chem. 2014, 126, 5409.

(b) Liu, Z.; Liu, J.; Zhang, L.; Liao, P.; Song, J.; Bi, X. Angew. Chem., Int. Ed. 2014, 53, 5305.

(c) Liu, J.; Liu, Z.; Wu, N.; Liao, P.; Bi, X. Chem.-Eur. J. 2014, 20, 
2154.

(d) Fang, Z.; Liao, P.; Yang, Z.; Wang, Y.; Zhou, B.; Yang, Y.; Bi, X. Eur. J. Org. Chem. 2014, 5, 924.

(e) Fang, G.; Li, J.; Wang, Y.; Gou, M.; Liu, Q.; Li, X.; Bi, X. Org. Lett. 2013, 15, 4126.

(f) Liu, J.; Fang, Z.; Zhang, Q.; Liu, Q.; Bi, X. Angew. Chem., Int. Ed. 2013, 52, 6953. (g) Liu, Y.; Barry, B.-D.; Yu, H.; Liu, J.; Liao, P.; Bi, X. Org. Lett. 2013, 15, 2608.

(h) Fang, Z.; Yuan, H.; Liu, Y.; Tong, Z.; Li, H.; Yang, J.; Barry, B.-D.; Liu, J.; Liao, P.; Zhang, J.; Liu, Q.; Bi, X. Chem. Commun. 2012, 48, 8802 .

[16] Evano, G.; Blanchard, N.; Toumi, M. Chem. Rev. 2008, 108, 3054.

(Qin, X.) 